\title{
Multi-component strain development in superconducting magnet coils monitored using fibre Bragg grating sensors fabricated in highly linearly birefringent fibre
}

\author{
Edmon Chehura ${ }^{1}$, Stephen W James ${ }^{1}$, Adam Johnstone ${ }^{3}$, M'hamed Lakrimi ${ }^{3}$, Fred Domptail ${ }^{2}$, \\ Andrew Twin ${ }^{2}$ and Ralph P. Tatam ${ }^{1}$ \\ ${ }^{1}$ Department of Engineering Photonics, \\ School of Engineering, \\ Cranfield University, Bedfordshire MK43 0AL, \\ Bedford, United Kingdom \\ ${ }^{2}$ Oxford Instruments, \\ Tubney Woods, Abingdon, \\ Oxon OX13 5QX, United Kingdom \\ ${ }^{3}$ Siemens Magnet Technology Ltd, \\ Wharf Road Eynsham, Witney, \\ Oxfordshire OX29 4BP, United Kingdom
}

\begin{abstract}
The commissioning of superconducting magnet coils was monitored using embedded optical fibre Bragg grating sensors (FBG) fabricated in highly linearly birefringent (HiBi) fibre. The HiBi FBG sensors monitored the internal strain developed in the coils during the energisation of the coils. The development of multiple components of strain in the coils when the magnet was energised and quenched was monitored, revealing phenomena that it had not been previously possible to measure using other sensor technologies.
\end{abstract}

PACS: 42.81.Pa, 42.81.Gs, 07.60.Vg, 07.10.Pz

\section{Short title}

Multi-component strain in superconducting magnet coils monitored using HiBi FBGs

\section{Submitted to the Smart Materials and Structures}

\section{Introduction}

Superconducting magnets are used in applications that range from laboratory scale equipment to large scale energy storage systems. Nuclear magnetic resonance (NMR) and magnetic resonance imaging (MRI) are examples of applications in the medical field where they are used to visualise the internal structure and function of the body [1]. A superconducting magnet consists of a number of coaxial coils. The turns of individual coils are bound together and to a support/former by impregnating the structure with resin, providing high mechanical stability to prevent relative movement of the components of the coil in the presence of the magnetic field [2]. When the magnet is energised, large stresses are induced in the coil windings because of the Lorentz forces between the magnetic field and the current. These forces lead to large hoop stresses and to axial compression in the windings [2]. The construction of a superconducting magnet is very challenging as the residual resistance of the magnet has to be minimal. Superconducting characteristics, namely zero electrical resistance and the exclusion of the interior magnetic field (the Meissner effect), are exhibited when the 
temperature of the superconducting material is lowered below the critical temperature [3]. The superconducting magnet is therefore placed in a bath or cryostat containing a liquid coolant, for example helium at its normal boiling point.

A superconducting magnet will function properly if all of its conductors remain in the superconducting state. Heating will take place if electric current passes through any part of the windings that have become resistive. The heating effect has the tendency to increase the size of the non-superconducting zone which propagates rapidly into other parts of the magnet. The energy stored in the magnet is eventually dissipated, leading to the rapid evaporation of liquid helium. Under these circumstances the magnet will heat up to temperatures well above 4.2 K. This effect is referred to as a quench [2]. Microscopic defects within the magnet structure or microscopic movements of wires in the coils may be sufficient to initiate a quench [2].

It is in the interest of the manufacturers to produce very stable superconducting magnets that will withstand the large electromagnetic forces that exist during operation. The magnet should not quench unnecessarily both during post-production testing and during its in-service life, for economic reasons that are dictated by the cost of liquid helium. Efficient production and operation of superconducting magnets will benefit from advanced sensors that may be embedded within the fabric of the magnet structure without compromising its structural integrity. The sensors are required to monitor the development of internal strain during the processes involved in the production of the magnet and throughout the test and training schedule of the magnet. Currently, sensors are not deployed in the coil structure for a number of reasons which range from the problem of intrusion to that of electromagnetic interference if the sensors are electrical in nature. Optical fibre sensors have however demonstrated the requisite properties for these applications [4-9]. The feasibility of the use of optical fibre Bragg gratings (FBGs) to measure temperature $[4,5]$ and a single strain [6-8] component at cryogenic temperatures has been investigated previously using FBGs fabricated in single mode (SM) fibre. The strain experienced by the magnet is however, multi-component in nature due to the Lorentz forces which operate within the solenoid and between solenoids in the case of a split-pair magnet. These forces are responsible for the large hoop, radial and axial strains that the magnet is known to exhibit [2]. The change in the reflection spectrum of an embedded optical fibre Bragg grating sensor will contain contributions from these forces. However, it is not possible to separate these contributions when the FBG is fabricated within a single mode optical fibre. FBGs fabricated in highly linearly birefringent (HiBi) fibre have been used previously to facilitate measurements in systems in which the sensor may experience a combination of transverse and axial loads [10].

This paper reports the first use of FBG sensors fabricated in HiBi fibre to monitor the development of multiple components of internal strain within the superconducting magnet coil during commissioning and during quench events. Two magnets were investigated which were representative of different production scales. The first was a small scale bespoke magnet designed for the research market, while the second was a large magnet that is used in medical imaging systems. The construction of the composite coils, as described in sections 4 and 5, are very different and therefore the intention of this work was to demonstrate that FBGs written in HiBi optical fibres are able to reveal phenomena occurring within the magnets that cannot be measured using other approaches rather than to compare phenomena occurring in each magnet.

\section{Principles of HiBi FBG sensors}

Two types of optical fibre which have been used previously as embedded sensors in composite material and in which it is possible to fabricate FBGs were considered for this work; singlemode (SM) and highly linearly birefringent (HiBi). SM fibre has an isotropic 
refractive index distribution in the core and supports one propagation mode of light above the cut-off wavelength. HiBi fibres are characterised by an anisotropic refractive index distribution within the core that is produced by, for example, introducing stress applying parts into the cladding of the fibre [11] (figure 1a). HiBi fibre exhibits two refractive indices along two orthogonal axes such that light coupled into the fibre propagates in two orthogonally polarised eigenmodes, with slightly different speeds, without cross-coupling between the modes. This intrinsic birefringence is generally larger than any externally introduced birefringence, for example from bending, twisting or transverse loading of the fibre, and therefore linearly polarised light coupled into a single eigenmode will propagate without change. Had SM fibre been used then the state of polarisation of the light propagating through the fibre would have been perturbed not only by the application of transverse loading on the FBG sensor but also by mechanical perturbations on the rest of the optical fibre embedded within the composite component, adding significant complexity to the recovered signal. The use of HiBi fibre enables the effective transverse strain to be recovered at each FBG sensor location, as described in detail below.

An FBG is a local periodic modulation of the refractive index within the core of an optical fibre imprinted into the core by exposure to the output from a UV laser using either a phase mask or an interferometer [12, 13]. Typical FBG lengths range from $0.1 \mathrm{~mm}$ to $10 \mathrm{~mm}$. When light from a broadband source is coupled into the fibre, the FBG reflects a narrow wavelength band of full-width half-maximum of the order of $0.1 \mathrm{~nm}$, centred at the Bragg wavelength. Measurands which interact with the fibre and alter the effective refractive index and/or the grating period will cause a shift in the reflected Bragg wavelength. A wide range of FBG sensor systems have been demonstrated for the measurement of parameters such as strain, temperature, and pressure [14-16]. When an FBG is fabricated in HiBi fibre, two Bragg peaks with centre wavelengths that differ by typically $0.35 \mathrm{~nm}$ will exist simultaneously [11], one polarised along each of the orthogonal axes of the fibre (figure 1b). HiBi FBG sensors therefore provide the capability to measure orthogonal components of strain at the same location $[10,17,18]$. Equation 1 can be used to calculate the effective transverse strain measured by a single HiBi FBG (i.e. 2 Bragg peaks) [10]

$$
\varepsilon_{f}-\varepsilon_{s}=\frac{2}{n_{0}{ }^{2}\left(p_{12}-p_{11}\right)}\left[\frac{\Delta \lambda_{f}}{\lambda_{B, f}}-\frac{\Delta \lambda_{s}}{\lambda_{B, s}}\right]
$$

In this equation, $\varepsilon_{f}$ and $\varepsilon_{s}$ are transverse strains along the fast $(f)$ and slow $(s)$ axes of the fibre respectively, $\lambda_{B, i}(i=s, f)$, are the two Bragg peak wavelengths at quiescent conditions, $\Delta \lambda_{s}$ and $\Delta \lambda_{f}$, are the wavelength shifts of the Bragg peaks, $n_{0}=1.4498$ is the average refractive index, and the constants $p_{11}=0.113$ and $p_{12}=0.251$ are the Pockels strain-optic coefficients [19].

For all the results presented the effective transverse strain was calculated by subtracting the in-plane wavelength component from the out-of-plane component for each HiBi FBG using equation 1 .

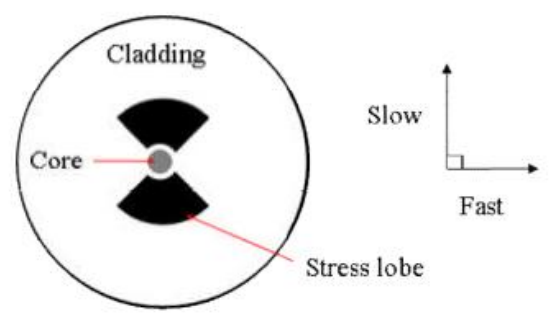

(a)

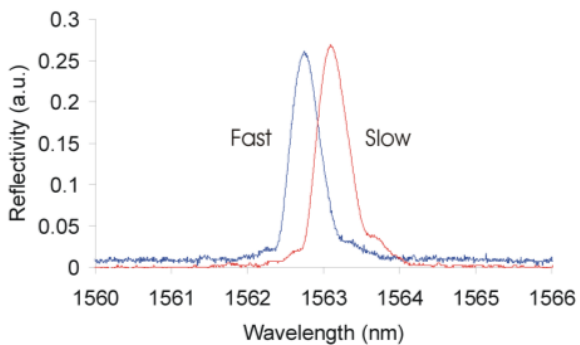

(b)

Figure 1: (a) The cross-section of a HiBi fibre with bow-tie stress rods and (b) reflection spectrum from an FBG fabricated in this type of fibre. 
Optical fibres exhibit a weak Verdet constant, and the magnetic field produced by the coil could therefore perturb the FBG spectrum via the Faraday Effect [20]. However, the FBG sensors are mounted on the top surface of the coil and therefore the effect of the magnetic field, which is aligned along the axis of the coil, is too small to influence the measured Bragg wavelength shift of the FBG sensors orientated around the circumference (hoop) direction [21].

\section{Experimental}

HiBi FBG sensors that were embedded within the magnet structure were used to measure strain developed within composite superconducting magnet coils manufactured by Oxford Instruments (OI) and Siemens Magnet Technology (SMT) during commissioning. The OI magnet system consisted of steel over-bind reinforcement, which introduces a compression of the order of 100MPa, whereas the SMT magnet was not reinforced. The HiBi FBG sensors were located midway through the cross section of the OI magnet system between the superconducting windings and the stainless steel over-bind reinforcement on the outside. The sensors in the SMT magnet system were located on the outside surface of the solenoid but within layers of glass fibre cloth. The development of multi-strain components in the two types of superconducting magnet coils was monitored during energisation and when the magnet underwent quenching, which are the normal testing and training schedules for commissioning new superconducting magnets.

Two wavelength-division-multiplexed (WDM) HiBi FBGs were installed in each of the superconducting magnets. The gratings were fabricated at the wavelengths of $1564 \mathrm{~nm}$ and $1572 \mathrm{~nm}$, respectively, each with a reflectivity of about $60 \%$. The HiBi fibre (Fibercore HB1500) consisted of bow-tie stress lobes (figure 1a). The fibre had core and cladding diameters of $8 \mu \mathrm{m}$ and $125 \mu \mathrm{m}$, respectively, while a protective polyacrylate buffer coating brought the total diameter of the optical fibre to $242 \mu \mathrm{m}$. It should be noted that, prior to FBG inscription, the buffer coating was removed from each of the $5 \mathrm{~mm}$ long sections of fibre to be exposed to the UV radiation, and that the FBGs were not re-coated. Each HiBi FBG sensor was embedded such that its slow and fast polarisation axes were aligned with the surface and the radial directions of the coil, respectively. The polarisation axes of the HiBi fibre were identified through observation of the diffraction pattern from the fibre when illuminated from the side with a Helium-Neon laser source $(633 \mathrm{~nm})$ as the fibre was rotated [22]. The polarisation axes of the fibre were then marked with scotch tape. The resolution of the rotary stages was $2^{\circ}$. Double-sided Kapton tape was attached to the surface of the magnet to provide anchor locations either side of each HiBi FBG to aid the alignment of its polarisation axes with the coil and to route the fibre over the surface of the magnet. The adhesion of the fibre to the structure was ultimately done by the resin infused into the coil (section 4).

The wavelengths reflected by the HiBi FBG sensors in each polarisation eigenmode were measured independently and simultaneously using a custom designed interrogation system [23] based on a scanning fibre Fabry-Perot (FFP) interferometer coupled to HiBi fibre (figure 2). The system, which operates around $1550 \mathrm{~nm}$, is capable of the simultaneous interrogation of WDM sensors, separating the orthogonally polarised components of the spectra $[11,23]$. The interrogation system has a maximum scan rate of approximately $250 \mathrm{~Hz}$ and a wavelength resolution of $40 \mathrm{pm}$. The interrogation system is equipped with a cell comprised of a mixture of hydrogen cyanide ( $\mathrm{HCN}$ ) and carbon monoxide (CO) gases, which is used for referencing spectra in the wavelength range from $1515 \mathrm{~nm}$ to $1595 \mathrm{~nm}$ (figure 2). 


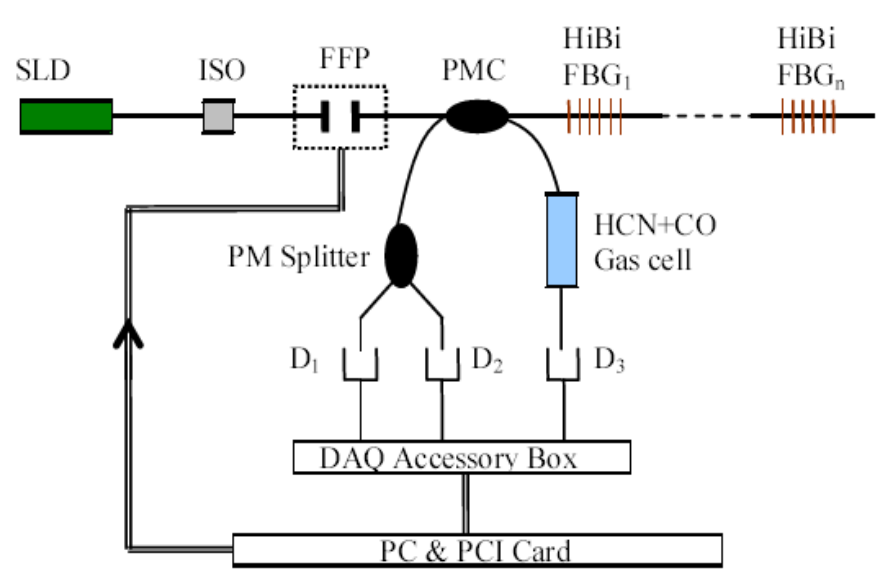

Figure 2: Schematic of the interrogation system. SLD: super luminescent diode; FFP: scanning HiBi fibre coupled Fabry-Perot tuneable filter; PM Splitter: fibre optic polarisation maintaining splitter; DAQ: data acquisition; $\mathrm{D}_{\mathrm{n}}$ : detectors; ISO: fibre optic coupled Faraday isolator; PMC: polarisation maintaining fibre optic coupler.

\section{OI magnet system}

The internal diameter and length of the prototype superconducting magnet that was investigated were $120 \mathrm{~mm}$ and $60 \mathrm{~mm}$ respectively. Superconducting wire was wound onto a metallic former using a machine that maintained constant strain and ensured tight binding from turn to turn and layer to layer. Glass fibre cloth was then wound on top of the coil (figures $3 a$ and $3 b$ ).

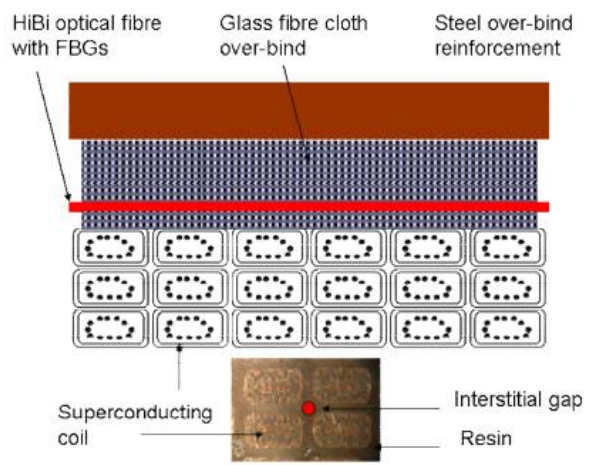

(a)

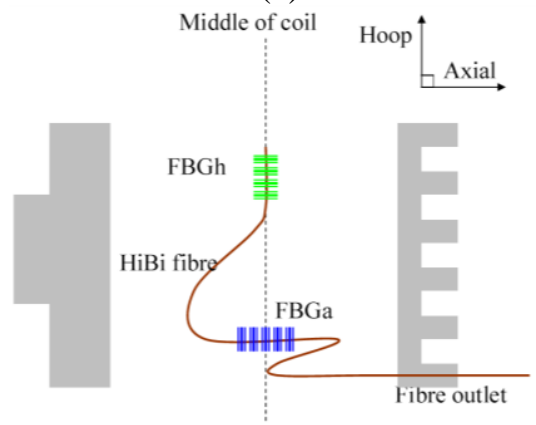

(c)

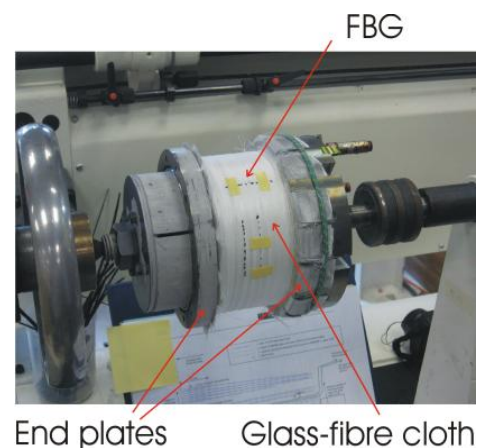

(b)

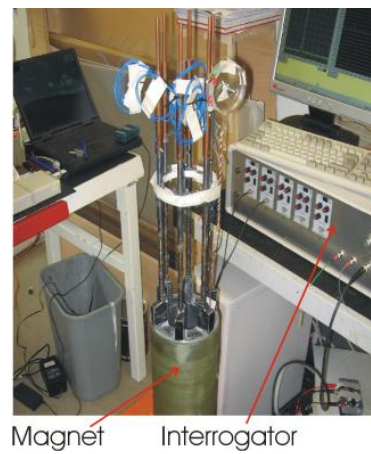

(d)

Figure 3: (a) Cross-section of the magnet, (b) Picture of the magnet showing sensors laid on the glass-fibre cloth, (c) Schematic diagram of the layout of the FBG sensors on a horizontal magnet as in (b), and (d) Picture of the magnet after potting. The slow axes of the HiBi fibres were laid in-plane while the fast axes lay out-of-plane to the surface of the coil. FBGa is along the axis of the coil while FBGh is along the circumference of the coil. The 2 FBGs are $9 \mathrm{~cm}$ apart. 
The HiBi FBG sensors were placed onto the surface of the glass fibre cloth according to the schematic diagram of figure $3 \mathrm{c}$. This procedure ensured that there was no direct contact between the superconducting wire and the bare fibre sensors thereby preventing potential mechanical damage to the sensors. It was ensured that, at the FBG sensor locations, the HiBi fibre was laid such that the slow axes lay in-plane (i.e. coil surface) while the fast axes lay out-of-plane (i.e. radially) to the surface of the coil. The sensors were all laid halfway between the end plates such that one HiBi FBG lay parallel to the axis of the magnet (FBGa), referred to as the axial direction, and one along the circumferential (FBGh) direction of the magnet, referred to here as the hoop direction (figure 3c).

Glass fibre cloth was then wound onto the magnet to fill up the region of the magnet between the two end plates/caps of the metallic former. The completed magnet was vacuumimpregnated with resin and was cured in an autoclave that was maintained at a temperature of $80{ }^{\circ} \mathrm{C}$ for 12 hours to bind the coils, glass fibre cloth and the sensors together. The surface of the magnet was, after potting, machined and fitted with steel over-bind for reinforcement (figure $3 \mathrm{a}$ ). Figure $3 \mathrm{~d}$ shows the magnet after potting. The superconducting magnet was mounted concentrically inside another magnet that was required for providing a background magnetic field to the superconducting coil. The magnet system was placed in a cryostat and cooled down from room temperature to $4.2 \mathrm{~K}$ using liquid helium. The sensors were connected to the interrogation system via a feed-through at the top of the cryostat.

\subsection{Coil energisation}

With the coil cooled to $4.2 \mathrm{~K}$, the outer magnet was energised and the current maintained at 100 A to provide the background magnetic field to the inner superconducting magnet coil. Data acquisition was started when the inner superconducting coil was energised. The superconducting coil was energised to $100 \mathrm{~A}$ at a rate of $5 \mathrm{~A}$ /minute (taking $\sim 21$ minutes) followed by an increased energising rate of $10 \mathrm{~A} /$ minute to $200 \mathrm{~A}$ (up to $\sim 34$ minutes) before de-energising to $0 \mathrm{~A}$ at a rate of $10 \mathrm{~A}$ /minute (figure 4 ).

The Bragg wavelength shift is well correlated with the profile of the energisation current (figures $4 \mathrm{a}$ and $4 \mathrm{~b}$ ) but exhibit oscillations during both the energisation and de-energisation processes. FBGa, which was aligned with the axial direction of the coil, has an opposite Bragg wavelength response to that of FBGh, which was aligned with the hoop direction of the coil. This behaviour suggests that the expansion of the coil in the hoop direction is accompanied by a simultaneous contraction of the coil in the axial direction. The maximum wavelength shift exhibited by FBGh was $\sim 0.9 \mathrm{~nm}$ (figure 4a), which is approximately three times that recorded by FBGa (figure 4b), indicating that greater strain is measured in the hoop than in the axial direction on the coil.

Figure $4 \mathrm{c}$ shows the effective transverse strains developed in the coil during energisation. Oscillations of approximately $1200 \mu \varepsilon$ peak-to-peak amplitude are exhibited by the transverse strain measured by FBGh, which was aligned with the hoop direction of the coil. FBGa, which was aligned with the axial direction of the coil, measured transverse strain oscillations of approximately $700 \mu \varepsilon$ peak-to-peak amplitude. It may be inferred from the oscillations in the transverse strain measurements that energisation and de-energisation induce local mechanical oscillations in the coil. 


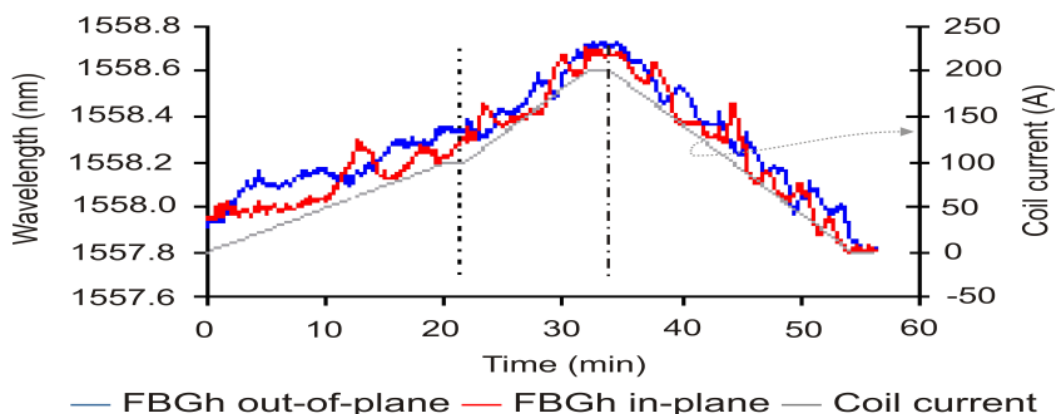

(a)

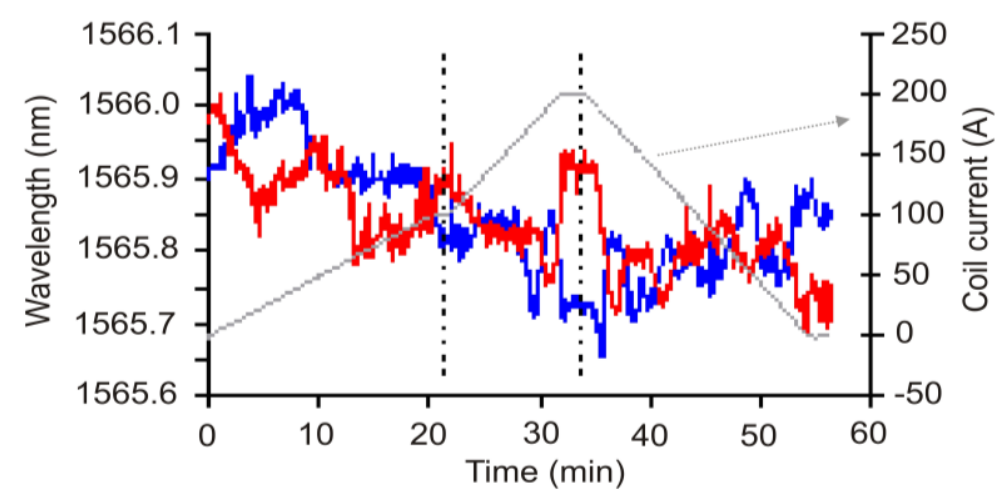

— FBGa out-of-plane — FBGa in-plane - Coil current

(b)

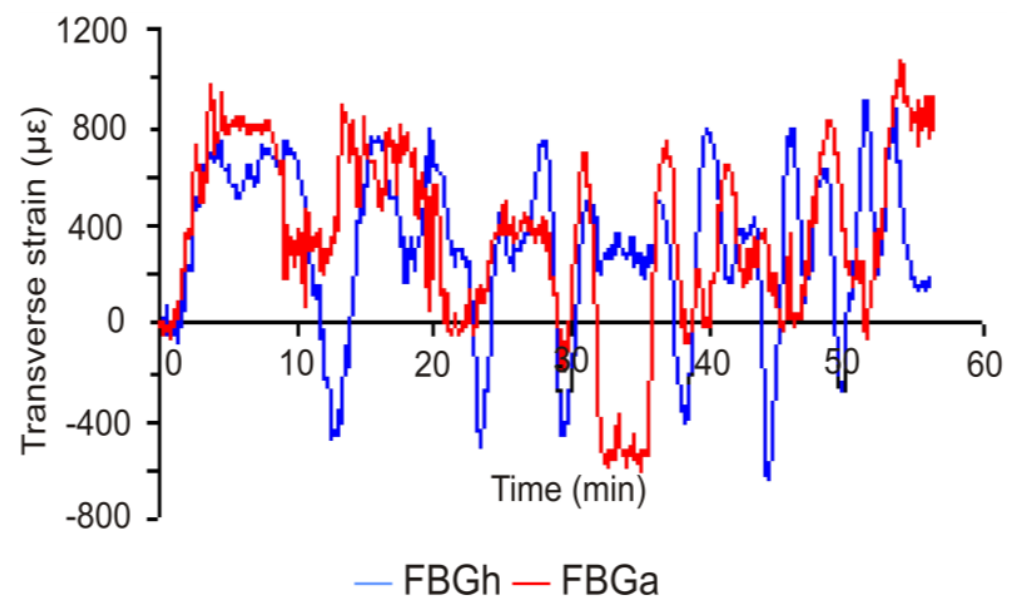

(c)

Figure 4: The response of the Bragg wavelengths of the HiBi FBG to energisation for (a) FBGh aligned with the hoop and (b) FBGa aligned with the axial directions of the coil. The vertical dashed lines indicate the time when the energisation rate was altered. (c) Effective transverse strains calculated from the wavelength shifts of figures $4 a$ and $b$, by subtracting the in-plane from the out-of-plane component using equation 1. 


\subsection{Energisation leading to spontaneous quench}

The outer magnet was energised, at a rate of $2 \mathrm{~A}$ /minute, to $110 \mathrm{~A}$ and the current was then maintained at this value. The inner superconducting coil was energised to $200 \mathrm{~A}$ at a rate of $20 \mathrm{~A} /$ minute (up to $\sim 10$ minutes) followed by further energisation at a rate of $10 \mathrm{~A} /$ minute to a target value of $400 \mathrm{~A}$ (figures $5 \mathrm{a}$ and $5 \mathrm{~b}$ ). However, the intended target of $400 \mathrm{~A}$ was not reached as the coil quenched at $211 \mathrm{~A}$, after approximately 13 minutes of energisation (figures $5 \mathrm{a}$ and $5 \mathrm{~b}$ ). The quench is referred to as a spontaneous quench.

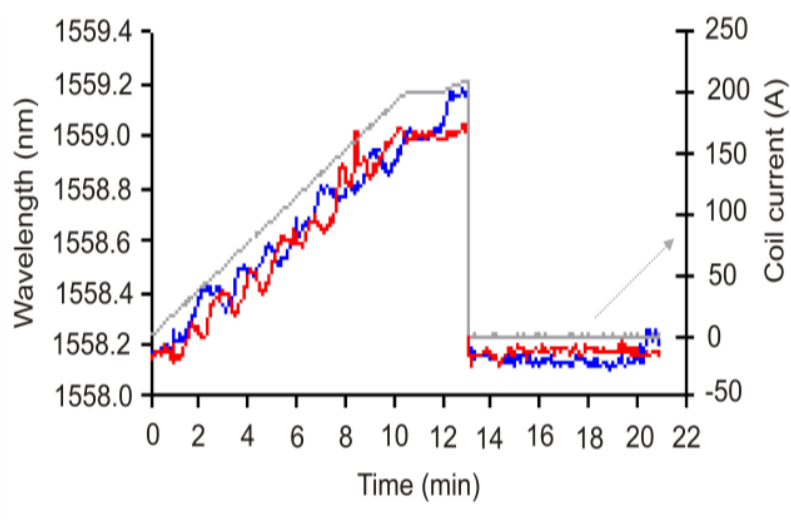

- FBGh out-of-plane - FBGh in-plane - Coil current

(a)

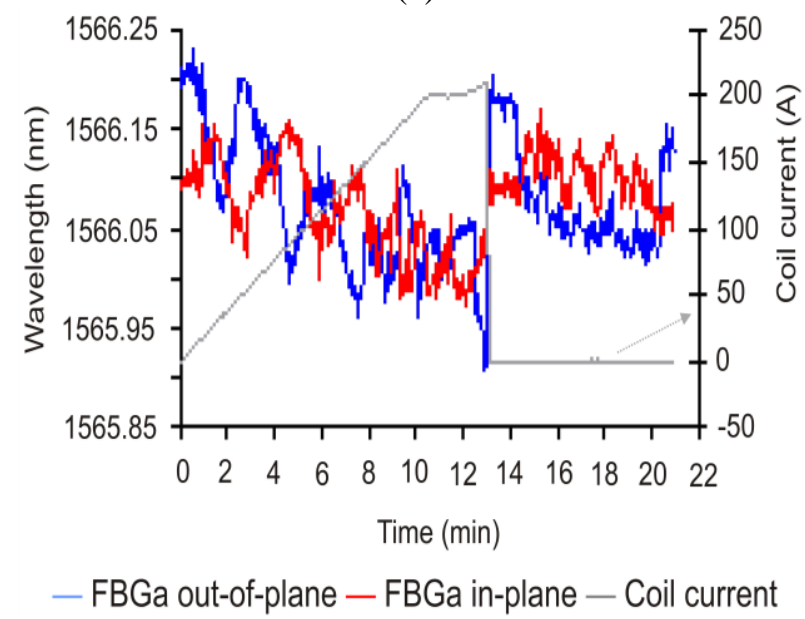

(b)

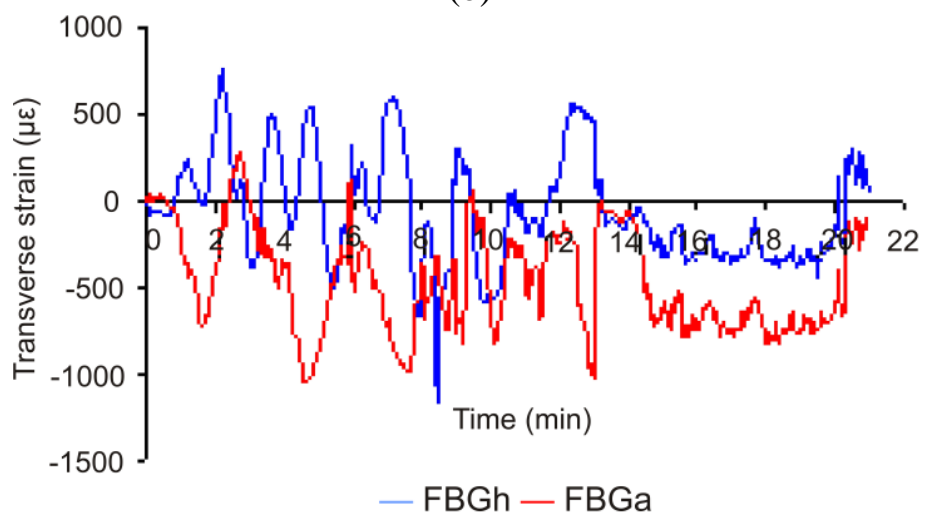

(c)

Figure 5: The response of the Bragg wavelengths of the HiBi FBGs to energisation for (a) FBGh aligned with the hoop and (b) FBGa aligned with the axial directions of the coil. (c)

Effective transverse strains. 
The energisation process was monitored by FBGh and FBGa which were laid in the coil such that they were aligned with the hoop and axial directions of the coil respectively. The wavelength shifts of FBGh (figure 5a) are opposite to the wavelength shifts of FBGa (figure 5b). This behaviour is expected given that, when the coil expands in the hoop direction it is accompanied by a concomitant contraction in the axial direction. The wavelength shifts exhibited oscillations. The spontaneous quench is depicted by the sudden change in the wavelength shift of the FBG sensors in figures $5 \mathrm{a}$ and $5 \mathrm{~b}$ after about 13 minutes of energisation. The wavelength shift is well correlated with the profile of the energising current. The maximum wavelength shift exhibited by FBGh was $\sim 1 \mathrm{~nm}$ (figure $5 \mathrm{a}$ ), which is approximately three times that recorded by FBGa (figure 5b), indicating that greater strain is measured in the hoop direction on the coil.

The effective transverse strains are shown in figure $5 \mathrm{c}$. The transverse strain measured by FBGh (aligned with the hoop) is largely in anti-phase with the transverse strain measured by FBGa (aligned with the axial direction) up to the onset of the spontaneous quench. Transverse strain oscillations with peak-to-peak amplitudes of approximately $700 \mu \varepsilon$ can be observed before the onset of the spontaneous quench, after which the oscillations reduce in amplitude to a peak-to-peak value of about $100 \mu \varepsilon$ (figure $5 \mathrm{c}$ ). The strain oscillations have not been observed previously when strain gauges were mounted on over-bound superconducting magnet coils. It is possible that the steel over-bind acted as a buffer to the strain oscillations which were experienced by the superconducting coils to such an extent that the strain gauges mounted on the over-bind surface did not have enough sensitivity to detect them.. In the case of these measurements, the sensors are embedded within glass fibre cloth which is attached to the surface of the magnet to which steel over-bind reinforcement is then attached. It is possible that the sensors oriented with the circumferential direction of the magnet will lie in the grooves formed between the superconducting coils while those sensors oriented with the axial direction of the magnet will cross the coil winding. Global or local movements of the wire turns could result in oscillatory increases and decreases of the hoop strain and corresponding decreases and increases of the axial strain. Measurements reported previously by Willsch et.al.[7], which used FBG sensors fabricated in single mode fibre (SM FBGs) that were directly bonded to the surface of the superconducting coils, did not show any oscillations in strain during energisation. However, oscillations were observed after the quench event and lasted for approximately 250s

\subsection{Energisation followed by forced quench}

The outer magnet was energised and maintained at a current of $110 \mathrm{~A}$ after which the inner superconducting coil was energised to $100 \mathrm{~A}$. A forced quench was then induced via a trigger that allowed current to pass through a resistive element built into the outer coil, leading to local heating in the coil. Figure 6a shows the two orthogonal components of the wavelength response from HiBi FBGh, which was aligned with the hoop direction of the superconducting coil. The profile of the energising electrical current is also plotted in the figure. Figure $6 \mathrm{~b}$ is a similar representation for $\mathrm{HiBi} \mathrm{FBGa}$ that was aligned with the axial direction of the superconducting coil. The onset of the quench is characterised by a sudden rise in the electrical current at approximately 2 seconds and this is illustrated in more detail in figures $6 \mathrm{c}$ and $6 \mathrm{~d}$ over a period of 5 seconds.

The wavelength responses of the FBG sensors are well correlated with the electrical current. The wavelength responses after the quench are characterised by oscillations whose period gradually lengthens. The in-plane wavelength component is in anti-phase with the out-ofplane wavelength component for any given HiBi FBG (figures 6a and 6b). FBGh, which was aligned with the hoop direction of the coil, exhibited a greater sensitivity to the quench than that of FBGa, which was aligned with the axial direction of the coil. The sudden increase in the wavelengths, followed by a sharp decrease during the quench (after $2 \mathrm{~s}$ ), as illustrated by 
figure $6 \mathrm{a}$, is opposite to the behaviour of figure $6 \mathrm{~b}$. The evolution of the strain components of the sensor embedded aligned with the hoop is therefore opposite to that aligned with the axial direction of the coil. The existence of oscillations in the wavelengths of the sensors immediately after a quench event was reported before but for SM FBG sensors [7].

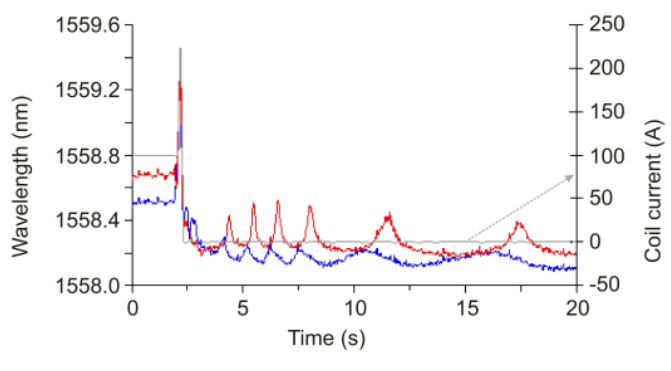

(a)

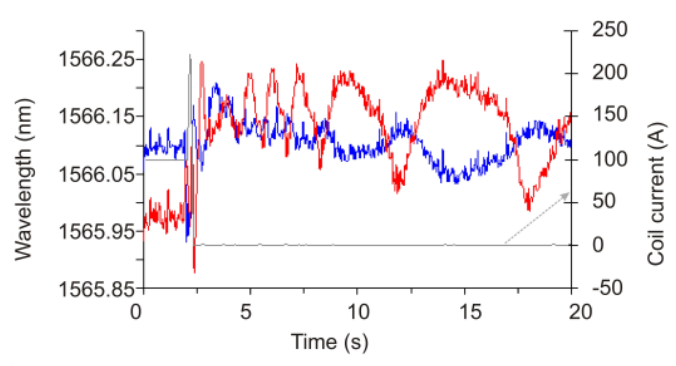

— FBGa out-of-plane — FBGa in-plane — Coil current

(b)

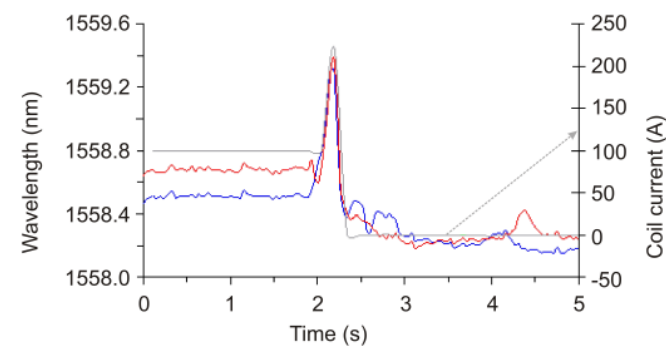

— FBGh out-of-plane - FBGh in-plane - Coil current

(c)

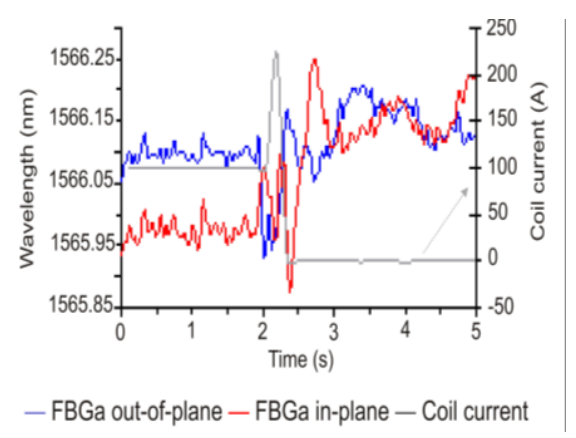

(d)

Figure 6: The wavelength response of the HiBi FBG to a forced quench for (a) FBGh aligned with the hoop and (b) FBGa aligned with the axial directions of the coil. (c) and (d) are magnifications of (a) and (b) respectively.

Figure 7 shows the effective transverse strains recorded by FBGh and FBGa, which were aligned with the hoop and axial directions of the coil respectively, following the forced quench. The signals show a complex oscillatory response, with the transverse strains having broadly similar responses, but with opposite signs. The period of the oscillations increase with increasing time after the quench. The delay between the oscillations observed for the two sensors suggests that the strains are induced by a travelling vibrational mode of the coil structure that was excited by the quench, as the two FBGs were physically separated by 90 $\mathrm{mm}$. The time delay between the sensors' responses is a constant proportion of the period of oscillation. Long term oscillations, lasting for over $200 \mathrm{~s}$, were observed within measurements made using non-birefringent single mode optical fibre in similar sized superconducting magnet systems [7]. However, those measurements were unable to resolve the axial and transverse strain components

. The oscillations suggest that the coil, either locally between the energised windings and the over-bind, or over the bulk of the structure, is set into mechanical oscillation after the quench process. It may be inferred from the strain oscillations that as the coil expands along the hoop direction it simultaneously contracts along the axial direction. Also plotted in figure 7 are the average hoop and axial strains measured by two resistance foil strain gauges (RFSGs) that were attached to the outer surface of the magnet, one aligned with the hoop and the other aligned with the axial directions of the coil. The hoop and axial strains from the RFSGs increased to a maximum of $2000 \mu \varepsilon$ and $1250 \mu \varepsilon$, respectively, at the onset of the quench before the strains decayed rapidly to approximately -480 and $-290 \mu \varepsilon$, respectively. The RFSG responses imply that the coil will finally settle at negative states of strain thus 
indicating that the magnet has dissipated energy as a result of the quench. The maximum transverse strain excursion from the HiBi FBG sensors is about $1500 \mu \varepsilon$, which is comparable to the maximum strains measured by the RFSGs. The RFSGs were, however, unable to record the oscillations in strain that were measured by the HiBi FBG sensors.

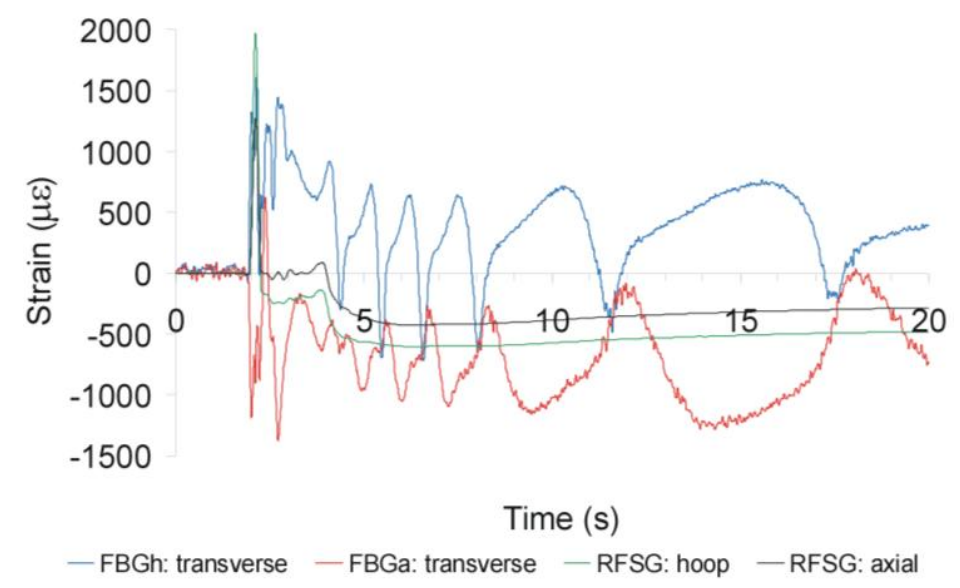

Figure 7: Effective transverse strains calculated from the wavelength shifts of figures 6a and $\mathrm{b}$ for FBGh and FBGa, respectively. The hoop and axial strains measured by resistance foil strain gauges (RFSG) are also plotted.

\section{SMT magnet system}

The prototype magnet that was investigated consisted of split-pair solenoids separated by 60 $\mathrm{mm}$ (figure 8). The internal diameter of the superconducting magnet was $291 \mathrm{~mm}$ and the width of each solenoid was $117 \mathrm{~mm}$. Superconducting wire was wound onto a metallic former using a machine that maintained constant strain and ensured tight binding from turn to turn and layer to layer. Glass fibre cloth was then wound on top of the coil (figures 8a and 8b). The HiBi FBG sensors were placed within the glass fibre cloth according to the schematic diagram of figure $8 \mathrm{c}$.

The laying and alignment of the polarisation axes of the HiBi FBG sensors in the superconducting coil was according to the configuration that was used for the OI magnet (section 4).. The superconducting magnet was not fitted with steel over-bind reinforcement. The resin system was the same as the one used with the OI magnet system and the same curing procedure was used. Figure $8 \mathrm{~d}$ shows a picture of the superconducting magnet after potting (i.e. encasing in resin). The magnet was cooled down to $4.2 \mathrm{~K}$. The test procedures to which the superconducting coil was subjected as part of the process of training the magnet were similar to those that were implemented for the OI magnet system. 


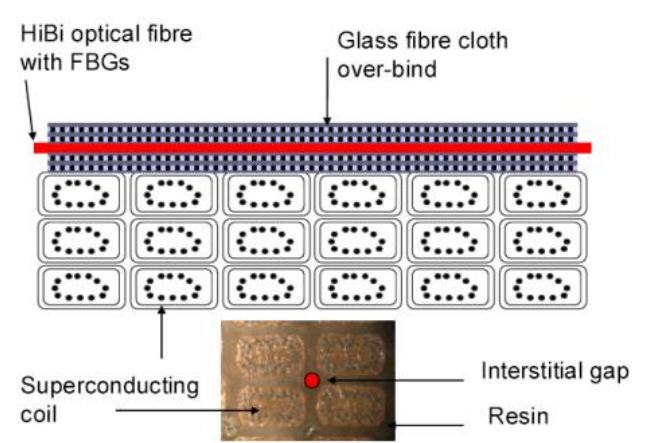

(a)

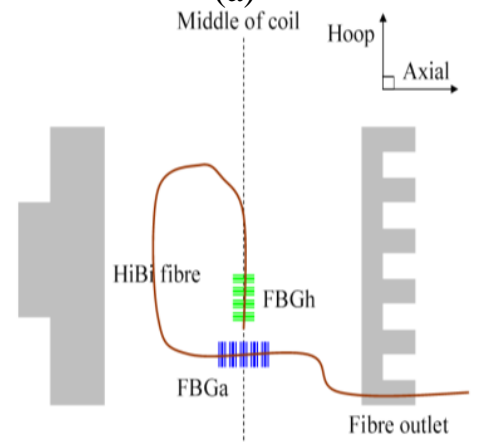

(c)

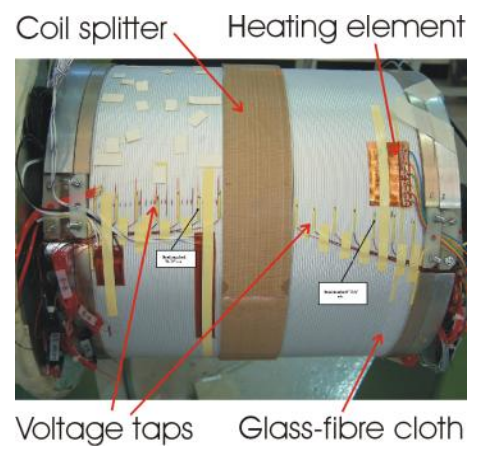

(b)

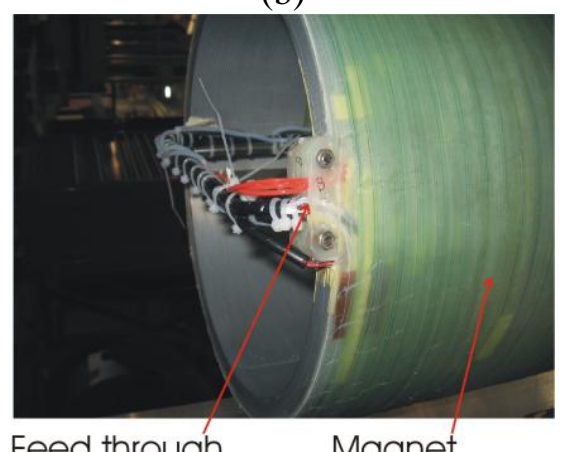

(d)

Figure 8: (a) Cross-section of the magnet, (b) Picture of the magnet showing sensors laid on the glass-fibre cloth, (c) Schematic diagram of the layout of the FBG sensors on a horizontal magnet as in (b), and (d) photograph of the magnet after potting. The slow axes of the HiBi fibres were laid in-plane while the fast axes lay out-of-plane to the surface of the coil. FBGa was along the axis of the coil while FBGh was along the hoop direction of the coil. The 2 FBGs are $9 \mathrm{~cm}$ apart.

\subsection{Coil Energisation}

The superconducting coil was energised to a target current of $350 \mathrm{~A}$ at a rate of $100 \mathrm{~A} /$ minute. The behaviour of the coil during the energisation process was studied using the HiBi FBG sensors embedded into the coil. Figures $9 \mathrm{a}$ and $9 \mathrm{~b}$ show the wavelength shifts of two HiBi FBG sensors aligned with the hoop and axial directions of the coil, along with the profile of the energising current. In Figure 9 (a), the wavelength shifts exhibited by the Bragg reflections from the eignaxes of FBGh, which was aligned with the hoop direction of the coil, were characterised by oscillations, with the out-of-plane component showing larger amplitude. The signals did not show a change in their average value as the current increased. The period of the oscillations decreased from about 50s initially, to $\sim 8 \mathrm{~s}$ after $200 \mathrm{~s}$ of energisation. The oscillations of the in-plane wavelength component are in anti-phase with the oscillations in the out-of-plane component. The wavelength shifts exhibited by the reflections from the eigenaxes of FBGa, shown in figure 9 (b), which was aligned with the axial direction of the coil, decrease as the current increases and the out-of-plane component exhibits oscillations of the same period variation as for FBGh. The in-plane wavelength component is noisier, but appears to exhibit a lower amplitude oscillation that is in phase with the out-ofplane component.

The effective transverse strains experienced by the two FBGs, determined by processing the data in figures 9(a) and 9(b), are shown in figure 9c. The effective transverse strain measured by FBGa, which was aligned with the axial direction of the coil appears to exhibit a low amplitude oscillation, similar to that which characterises the effective transverse strain measured by FBGh, which was aligned with the hoop direction of the coil. Both the amplitude and period of the oscillations decreased gradually as the energising current was increased. 
Such oscillations were not observed by the resistive foil strain gauges that were mounted on the over-bound superconducting magnet coil to monitor energisation. The effect is pronounced in this magnet because the $5 \mathrm{~mm}$ rectangular superconducting wire requires a larger turn radius. For an FBG sensor aligned with the hoop direction of the coil and located between turns that move apart locally, the FBG would record a corresponding decrease in the hoop strain. The small amplitude of the oscillations in the transverse strain measured by the sensor aligned with the axial direction of the coil may be attributed to the absence of steel over-bind reinforcement.

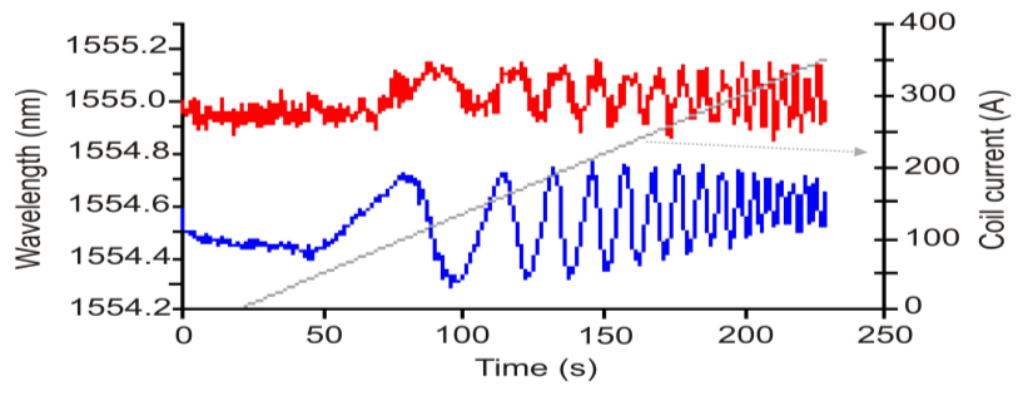

— FBGh out-of-plane — FBGh in-plane — Coil current

(a)

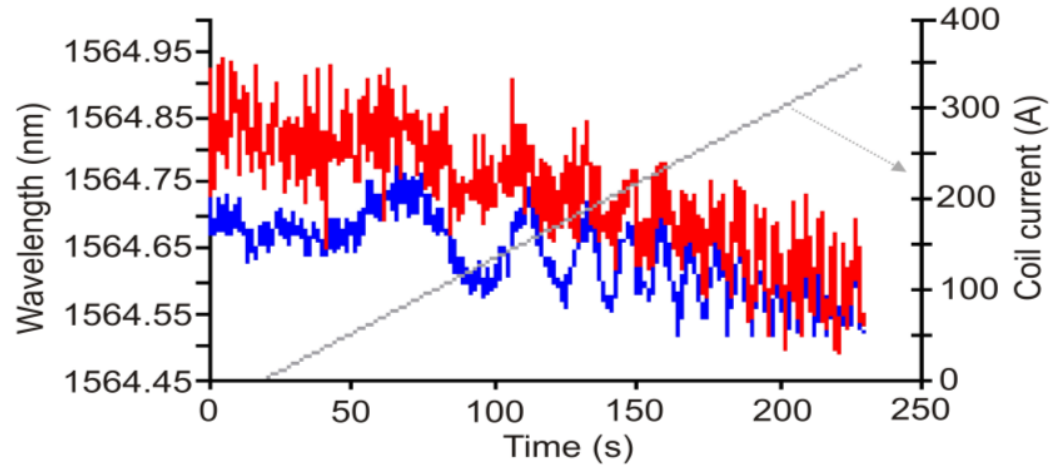

- FBGa out-of-plane — FBGa in-plane - Coil current

(b)

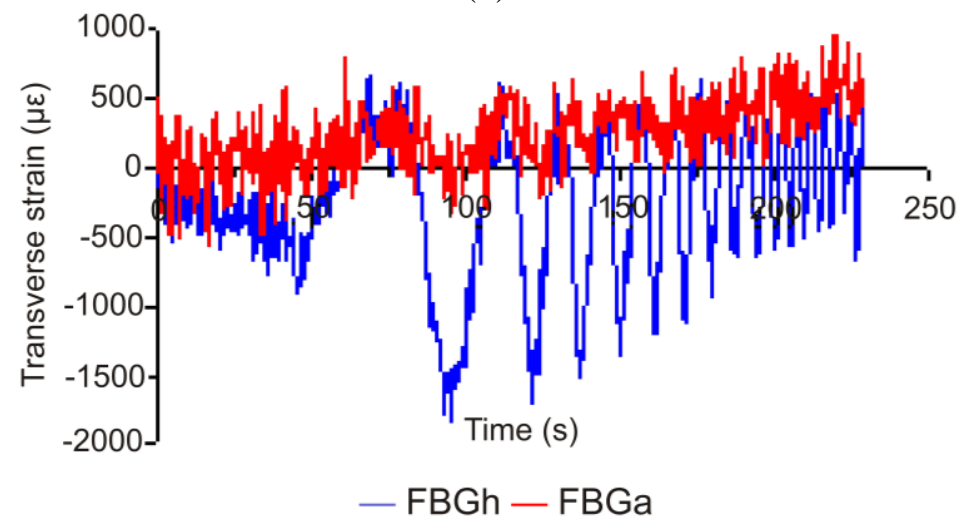

(c)

Figure 9: The response of the HiBi FBG wavelength to energisation for (a) FBGh aligned with the hoop and (b) FBGa aligned with the axial directions of the coil. (c) Effective transverse strains. 
The superconducting coil was energised to a target current of $350 \mathrm{~A}$ at a rate of $100 \mathrm{~A} /$ minute. A forced quench was then induced via a trigger that allowed current to pass through a resistive element built into the coil, which caused local heating in the coil. The quench was monitored using the HiBi FBG sensors embedded into the coil. Figures 10a and 10b show the wavelength shifts of $2 \mathrm{HiBi}$ FBGs which were aligned with the hoop and axial directions of the coil. Plotted alongside the wavelength shifts is the temperature of the coil measured by carbon ceramic temperature sensors. The onset of the forced quench is characterised by a sudden rise in temperature occurring approximately 3 seconds after the start of data logging.

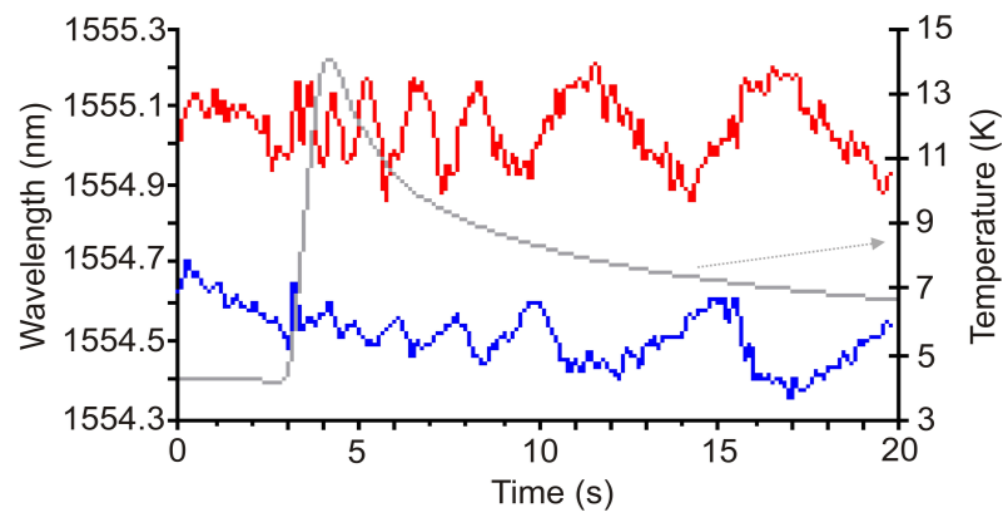

— FBGh out-of-plane — FBGh in-plane — Temperature

(a)

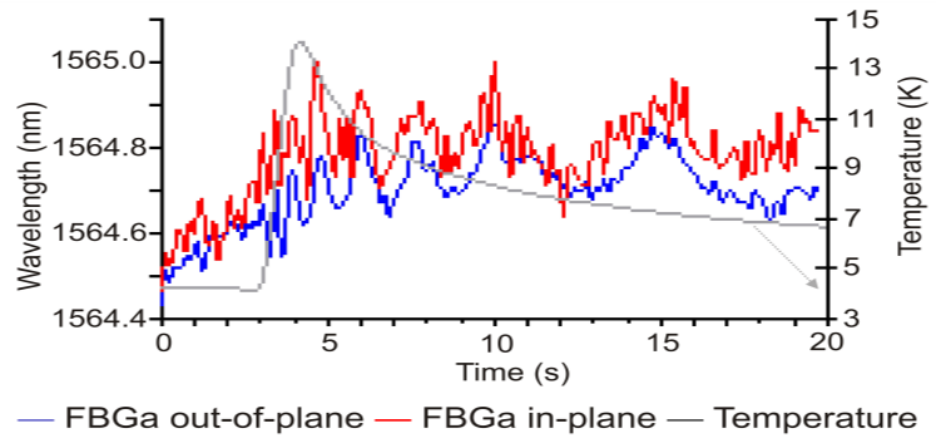

(a)

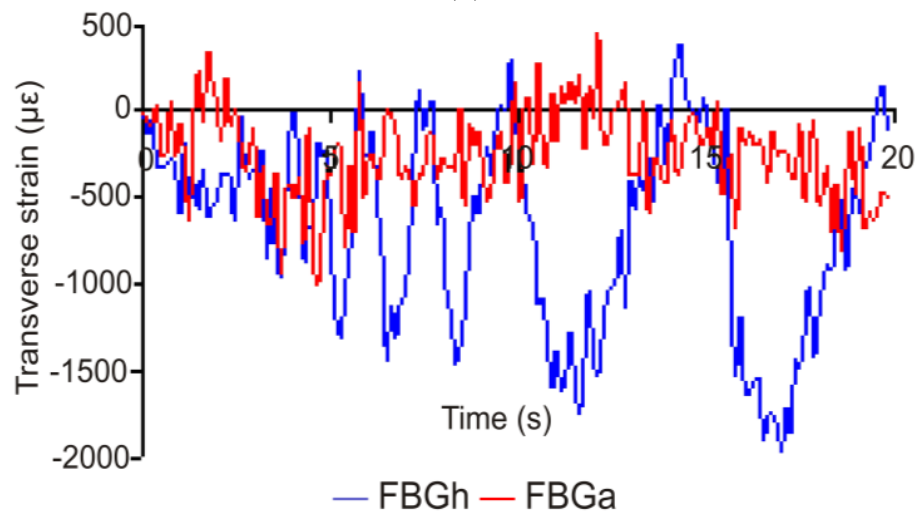

(c)

Figure 10: The response of the wavelength of the HiBi FBG to a forced quench for (a) FBGh aligned with the hoop and (b) FBGa aligned with the axial directions of the coil. (c) Effective transverse strains.

The wavelength shifts of FBGh, which was aligned with the hoop direction of the coil, exhibited oscillations from the onset of the quench. The period and amplitude of the oscillations gradually increased with time. The out-of-plane wavelength component of FBGh is in anti-phase with the in-plane component (figure 10a). The wavelength shifts of FBGa, 
which was aligned with the axial direction of the coil, exhibited an increase in wavelength shift from the onset of the quench until 5 seconds after the start of the data logging (figure $10 \mathrm{~b})$. The response of the Bragg wavelengths to the quench is again characterised by oscillations [7]. The period of the oscillations gradually increased over the duration of the measurement. The out-of-plane wavelength component of FBGa is in-phase with the in-plane component.

The effective transverse strain for each HiBi FBG is shown in figure 10c. The transverse strain measured by FBGa, which was aligned with the axial direction of the coil, appears not | to exhibit_periodic oscillations, unlike the transverse strain measured by FBGh, which was aligned with the hoop direction of the coil. The period and amplitude of the oscillations exhibited by FBGh gradually increased with time. This strain response is not expected to be permanent but that the oscillations will eventually disappear when the magnet return to equilibrium [7]. The time duration in which the quench event was monitored was chosen arbitrarily as the settling time of the magnet after the quench was not known prior to the experiment. The processing of the data was carried out after the experiment which meant that the strain profile could not be known during the experiment.

\section{Discussion}

The most significant difference between the two magnet systems investigated is that the OI magnet incorporated steel over-bind reinforcement while the SMT magnet did not have any reinforcement. The SMT magnet was larger than the OI magnet (i.e. $~ 2.5$ times larger for the internal diameter and $\sim 5$ times larger for the length) and also had split solenoids unlike the OI magnet. The HiBi FBG sensors were within the structure of the coil such that they were effectively located in the middle of the thickness of the magnet for the OI magnet and on the outer surface of SMT magnet. Transverse strains measured in both magnets during the energisation and quench processes were generally characterised by oscillations leading us to believe that these were induced by either global or local mechanical oscillations rather than by local delamination or failure of the composite material. Transverse strains measured by HiBi FBGs aligned with the hoop and axial directions of the coil were both characterised by oscillations for the OI magnet whereas the transverse strain measured by the HiBi FBG aligned with the axial direction exhibited oscillations that were of much smaller amplitude than that of the HiBi FBG aligned with the hoop direction exhibited for the SMT magnet. The FBG sensors in these measurements lay across the superconducting wires that were forced against the rectangular winding of stainless steel over-bind for the OI magnet. The configuration for the SMT magnet was similar but without the outer stainless steel over-bind. Global or local movements of the wire turns, within the brittle epoxy-bonded structure, could result in oscillatory behaviour in the hoop strain which is complemented by the oscillations in the axial strain. Thus the reduced oscillations in the transverse strain measured by the HiBi FBG aligned with the axial direction of the SMT magnet can be attributed to the absence of the reinforcement on the outside of the magnet. Strains measured in the reinforced (OI) magnet were generally greater than those measured in the un-reinforced (SMT) magnet. The transverse strains for sensors aligned with the hoop and axial directions of the reinforced (OI) magnet were characterised by oscillations that were in anti-phase. This behaviour suggests that when the superconducting coil is energised or quenched it experiences an expansion and contraction simultaneously in the hoop and axial directions respectively, at least at the location of the sensor. The quench causes the magnetic field produced by the coil to rapidly break down which in turn causes the relaxation of the coil to its original dimensions. The oscillations in the transverse strain, which are exhibited in both coils after the onset of the quench, are characterised by a period that lengthens gradually with time, which suggests that the coil will eventually stop oscillating when it is fully relaxed to its original dimensions. 


\section{Conclusion}

FBG sensors fabricated in $\mathrm{HiBi}$ fibres embedded within the composite coil structure surrounding the coil of superconducting magnets have been used to monitor the development of orthogonal strain components during energisation and quench processes. The measurements show the existence of radial and axial oscillations in the strain, additional information previously undetected by FBG sensors fabricated in SM fibre [7]. The oscillations suggest that the coil either locally, at the location of the sensors between the energised windings and the over-bind, or over the bulk of the structure is set into mechanical oscillations after the quench process. The results demonstrate the suitability of HiBi FBG sensors as quality control tools during the manufacturing and commissioning of superconducting magnet coils.

\section{Acknowledgements}

This work was supported by the Engineering and Physical Sciences Research Council (UK) via Cranfield University's IMRC and industrial partners; GKN Aerospace, Siemens and Oxford Instruments.

\section{References}

1. Lauterbur P C 1973 Image formation by induced local interactions: examples employing nuclear magnetic resonance Nature 242 190-191.

2. Balshaw N H 2006 Practical Cryogenics: An Introduction to Laboratory Cryogenics Oxford instruments NanoScience Handbook.

3. Gallop J C 1990 SQUIDS, the Josephson Effects and Superconducting Electronics CRC Press ISBN 0750300515.

4. Roths J, Andrejevic G, Kuttler R and Suber M 2006 Calibration of fiber Bragg cryogenic temperature sensors Tech. Gig. $18^{\text {th }}$ Int. Conf. on Optical fibre Sensors, TuE81 ISBN 1-55752-816-0.

5. Lupi C, Felli F, Ippoliti L, Caponero M A, Ciotti M, Nardelli V and Paolozzi A 2005 Metal coating for enhancing the sensitivity of fibre Bragg grating sensors at cryogenic temperature Smart Mater. Struct. 14 N71-N76.

6. James S W, Tatam R P, Twin A and Noonan P 2002 Strain Response of Fibre Bragg Grating Sensors at Cryogenic Temperatures Meas. Sci. and Technol. 13 1535-1539.

7. Willsch M, Hertsch H, Bosselmann T, Oomen M, Ecke W, Latka I and Hofer H 2008 Fiber optical temperature and strain measurements for monitoring and quench detection of superconducting coils Proc. of SPIE 7004 70045G.

8. Ecke W, Latka I, Habisreuther T and Lingertat J 2007 Fiber-optic Bragg grating sensors for structural health monitoring at cryogenic temperatures Proc. SPIE $\mathbf{6 5 3 0}$ 653002.

9. James S W, Tatam R P, Twin A, Bateman R and Noonan P 2003 Cryogenic temperature response of fibre optic long period gratings Meas. Sci. and Technol. 14 1409-1411.

10. Chehura E, Skordos A A, Ye C-C, James S W, Partridge I K and Tatam R P 2005 Strain development in curing epoxy resin and glass fibre/epoxy composites monitored by fibre Bragg grating sensors in birefringent optical fibre Smart Mater. Struct. 14 354-362.

11. Chehura E, Ye C-C, Staines S E, James S W and Tatam R P 2004 Characterization of the response of fibre Bragg gratings fabricated in stress and geometrically induced high birefringence fibres to temperature and transverse load Smart Mater. Struct. 13 888-895. 
12. Meltz G, Morey W M and Glenn W H 1989 Formation of Bragg gratings in optical fibres by transverse holographic method Opt. Lett. 14 823-825.

13. Dockney M L, James S W and Tatam R P 1996 Fibre Bragg gratings fabricated using a wavelength tuneable laser source and a phase mask based interferometer Meas. Sci. Technol. 7 445-448.

14. Karalekas D, Cugnoni J and Botsis J 2009 Monitoring of hygrothermal ageing effects in an epoxy resin using FBG sensor: a methodology study Composite Sci. Technol. 69 507-514.

15. Frias C, Frazao O, Tavares S, Vieira A, Marques A T and Simoes J 2009 Mechanical characterization of bone cement using fiber Bragg grating sensors Materials and Design 30 1841-1844.

16. Chehura E, James S W and Tatam R P 2007 Temperature and strain discrimination using a single tilted fibre Bragg grating Opt. Comm. 275 344-347.

17. Udd E, Schulz W, Seim J, Haugse E, Trego A, Johnson P, Bennett T E, Nelson D and Makino A 2000 Multidimensional strain field measurements using fibre optic grating sensors Proc. SPIE 3986 255-262.

18. Prabhugoud M and Peters K 2007 Finite element analysis of multi-axis strain sensitivities of Bragg gratings in PM fibers J. Intel. Mater. Sys. Struct. 18 861-873.

19. Maier R R J, MacPherson W N, Barton J S, Jones J D C, McCulloch S and Burnell G 2004 Temperature dependence of the stress response of fibre Bragg gratings Meas. Sci. Technol. 15 1601-1606.

20. Kersey A D and Marrone, M J 1994 Fiber Bragg grating high-magnetic-field probe Proc. SPIE 2360 53-56.

21. Donati S, Annovazzi-Lodi V and Tambosso T 1988 Magneto-optical fibre sensors for electrical industry: analysis of performances IEE Proc. J. Optoelectronics 135372 382.

22. Pannell C N, Tatam R P, Jones J D C and Jackson D A 1988 A fibre-optic frequency shifter utilising travelling flexure waves in birefringent fibre $J$. Inst. of Electronic and Radio engineers supplement 58 S92-S98.

23. Ye C-C, Staines S E, James S W and Tatam R P 2002 A polarisation-maintaining fibre Bragg grating interrogation system for multi-axis strain sensing Meas. Sci. Technol. 13 1446-1449. 